\title{
Functional impact of a single-nucleotide polymorphism in the OPRD1 promoter region
}

\author{
Huiping Zhang ${ }^{1,2}$, Joel Gelernter ${ }^{1,2,3,4}$, Jeffrey R Gruen ${ }^{3,5}$, Henry R Kranzler ${ }^{6}$, Aryeh I Herman ${ }^{1,2}$
} and Arthur A Simen ${ }^{1}$

The $\delta$-opioid receptor mediates rewarding effects of many substances of abuse. We reported an increased frequency of the minor G-allele of single-nucleotide polymorphism (SNP) rs569356 (the only variant identified so far in the promoter region of the $\delta$-opioid receptor gene (OPRD1)) in subjects with opioid dependence. In this study, we examined the functional significance of this variant. OPRD1 promoter region harboring SNP rs569356 was amplified by PCR and inserted into a firefly luciferase reporter vector. HEK293 cells were co-transfected with these constructs and a renilla luciferase vector to control for transfection efficiency. Expression of firefly luciferase (driven by the OPRD1 promoter) was measured by a dual luciferase reporter assay and normalized by renilla luciferase expression. Moreover, alleles altering expression were further assessed for binding of human brain nuclear proteins by electrophoretic mobility shift assay (EMSA). The minor G-allele was associated with significantly greater expression levels of firefly luciferase than the major A-allele of SNP rs569356 ( $P=0.003)$. EMSA also showed specific gel shift bands, suggesting that SNP rs569356 is situated in the binding site of potential transcription factors. These results suggest that the minor G-allele of SNP rs569356 may enhance transcription factor binding and increase OPRD1 expression. Journal of Human Genetics (2010) 55, 278-284; doi:10.1038/jhg.2010.22; published online 19 March 2010

Keywords: dual luciferase reporter gene assay; electrophoretic mobility shift assay; $\delta$-opioid receptor gene; PCR; promoter polymorphism

\section{INTRODUCTION}

Opioid receptors (mainly $\mu-, \delta$ - and $\kappa$-receptors) in the endogenous opioid system regulate neuronal activity through neurotransmission or neuromodulation. ${ }^{1}$ There is mounting evidence that opioid receptors directly mediate reward, tolerance and dependence associated with opioids such as morphine and heroin. ${ }^{2,3}$ Opioid receptors are also indirectly involved in the reinforcing properties of non-opioid drugs (such as cocaine and alcohol) due to the intimate relationship between the opioid system and the mesolimbic dopamine system., ${ }^{4,5}$ The basal release of dopamine in the nucleus accumbens is modulated by the stimulatory $\mu$ - and $\delta$-opioid systems and the inhibitory -opioid system. ${ }^{4}$

Variation in $\mu-, \delta$ - and $\kappa$-opioid receptor genes may change the expression level or activity of opioid receptors, leading to increased risk for drug or alcohol dependence. Take the $\delta$-opioid receptor gene as an example. Animal studies have demonstrated that the $\delta$-opioid receptor is involved in addictive processes. When the mouse $\delta$-opioid receptor gene is knocked out (by deletion of exon 2), morphine tolerance in mice is lost as well. ${ }^{6,7}$ The human $\delta$-opioid receptor gene
(OPRD1, located at 1p36.1-p34.3) is the first of the human opioid receptor genes to be cloned. ${ }^{8}$ It has a relatively conserved coding sequence. Only two coding sequence polymorphisms, a non-synonymous single-nucleotide polymorphism (SNP) C80T (Cys27Phe or rs1042114) in exon land a silent SNP T921C (Gly307Gly or rs2234918) in exon 3, have been identified. ${ }^{9,10}$ A study by Mayer et al. ${ }^{9}$ demonstrated a significant association of SNP T921C and heroin dependence in a German population. However, follow-up studies in another German population ${ }^{11}$ and two Han Chinese Populations ${ }^{12,13}$ did not support the finding. Our recent study ${ }^{14}$ also did not show evidence of association between SNP T921C and heroin dependence in European Americans. Nevertheless, a highly significant association of the non-synonymous SNP C80T and opioid dependence was observed. In addition, the promoter SNP rs569356 (A/G) also showed a statistically significant association with opioid dependence by comparing both allele and genotype frequencies of the marker in cases and controls. The minor G-allele of SNP rs569356 was enriched in opioid-dependent European American cases compared with European American controls.

${ }^{1}$ Department of Psychiatry, Yale University School of Medicine, New Haven, CT, USA; ${ }^{2}$ VA Connecticut Healthcare System, West Haven, CT, USA; ${ }^{3}$ Department of Genetics, Yale University School of Medicine, New Haven, CT, USA; ${ }^{4}$ Department of Neurobiology, Yale University School of Medicine, New Haven, CT, USA; ${ }^{5}$ epartment of Pediatrics, Yale University School of Medicine, New Haven, CT, USA and ${ }^{6}$ Department of Psychiatry and Genetics and Developmental Biology, University of Connecticut School of Medicine, Farmington, CT, USA

Correspondence: Dr H Zhang, Division of Human Genetics, Department of Psychiatry, Yale University School of Medicine, VA Connecticut Healthcare System, Psychiatry 116A2, 950 Campbell Avenue, West Haven, CT 06516, USA.

E-mail: huiping.zhang@yale.edu

Received 29 November 2009; revised 19 February 2010; accepted 24 February 2010; published online 19 March 2010 


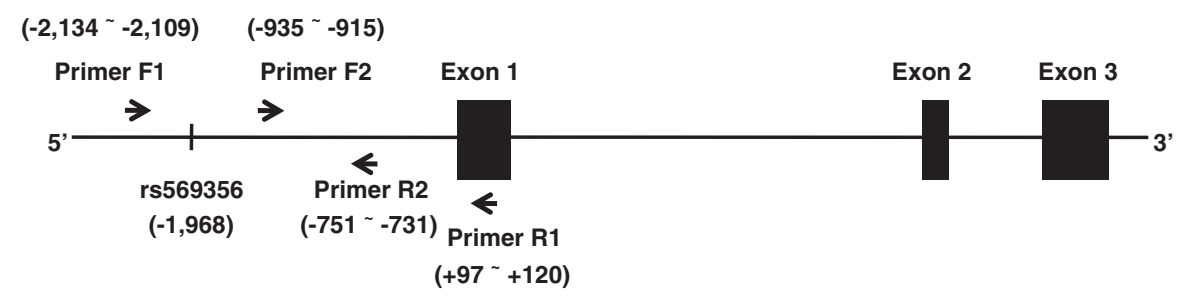

Figure 1 Schematic representation of the $\delta$-opioid receptor gene (OPRD1). Primers F1, F2, R2 and R1 are complementary to OPRD1 sequence at -2134 bp to $-2109 \mathrm{bp},-935 \mathrm{bp}$ to $-915 \mathrm{bp},-751 \mathrm{bp}$ to $-731 \mathrm{bp}$ and $+97 \mathrm{bp}$ to $+120 \mathrm{bp}$, respectively. SNP rs569356 (A/G) is located at $-1968 \mathrm{bp}$ in the promoter region of OPRD1.

The promoter SNP rs569356 is $1968 \mathrm{bp}$ upstream to the OPRD1 transcription start site and is the only documented variant identified in the promoter region of OPRD1 (refer to the NCBI SNP database http://www.ncbi.nlm.nih.gov/projects/SNP or the ABI SNPbrowser ${ }^{15}$ ). Polymorphisms located in promoter regions are presumed to affect gene transcription by modifying the coordinated action of multiple regulatory proteins through complex protein-DNA and proteinprotein interactions. ${ }^{16}$ Functional genetic variation in promoter region may alter the affinity of transcription factors and other modulatory proteins to bind to the DNA sequence and thus influence the specificity and kinetics of the transcription process. ${ }^{17-20}$ Although the promoter SNP rs569356 is 2289 bp apart from the non-synonymous SNP C80T, these two markers are in tight linkage disequilibrium $\left(r^{2}=0.833, \mathrm{D}^{\prime}=0.95\right) .{ }^{14}$ Therefore, it is necessary to clarify whether SNP rs569356 is truly an independent disease locus for opioid dependence or its positive association results were due to coinheritance of the non-synonymous SNP C80T.

The purpose of this study was to examine whether SNP rs569356 modulates OPRD1 transcription by changing the affinity of the promoter for transcription factors. We applied both the luciferase reporter gene assay and DNA-protein binding assay or electrophoretic mobility shift assay (EMSA) to examine the functional implication of OPRD1 promoter SNP rs569356.

\section{MATERIALS AND METHODS \\ DNA samples}

Genomic DNAs, extracted from peripheral blood cells of European American subjects, were included in our previous study regarding the association of $O P R D 1$ variants and drug or alcohol dependence. ${ }^{14}$ Subjects were recruited at the University of Connecticut Health Center and the VA Connecticut Healthcare System-West Haven campus, where the study protocol was approved by the respective institutional review boards. All subjects gave written informed consent before participating in the study.

Amplification of OPRD1 promoter region containing SNP rs569356 A pair of primers was designed to amplify $2250 \mathrm{bp}$ of OPRD1 promoter region containing SNP rs569356 by PCR. The forward primer (Primer F1: 5'-TGTGTGCCACCGTGCCCAGCCTTTT-3') was homologous to OPRD1 upstream sequence $(-2134 \mathrm{bp}$ to $-2109 \mathrm{bp}$ ) and the reverse primer (Primer R1: 5'-GCCCCGCTGTCTCTGCGCCTCGT-3') was complementary to part of the sequence ( $+97 \mathrm{bp}$ to $+120 \mathrm{bp}$ ) of OPRD1 exon 1. The PCR mixture contained $50 \mathrm{ng}$ of genomic DNA, $2.5 \mathrm{~mm}$ of each dNTPs, $200 \mathrm{~nm}$ of each primer, $2.5 \mu \mathrm{l}$ of dimethyl sulfoxide (Invitrogen, Carlsbad, CA, USA), $1 \times$ pfu Ultra II reaction buffer (Stratagene, La Jolla, CA, USA), and 2.5 units of pfu Ultra II fusion HS DNA polymerase (Stratagene) in a final volume of $50 \mu \mathrm{l}$. PCRs were run on a PTC-200 thermocycler (MJ Research, Waltham, MA, USA) and consisted of an initial denaturation step of $95^{\circ} \mathrm{C}$ for $2 \mathrm{~min}$, followed by 39 cycles of a two-step reaction $\left(95^{\circ} \mathrm{C}\right.$ for $30 \mathrm{~s}$ and $72^{\circ} \mathrm{C}$ for $\left.2 \mathrm{~min} 30 \mathrm{~s}\right)$.

\section{Construction of luciferase expression vectors}

PCR products were subcloned into pSMART HCAmp plasmid vector (1833 bp) (Lucigen, Middleton, WI, USA). The pSMART plasmid is a transcription-free vector optimized for large or otherwise difficult-to-clone DNA fragments. Briefly, the gel-purified PCR products were $5^{\prime}$ end phosphorylated by polynucleotide kinase (New England Biolabs, Ipswich, MA, USA) and ligated into pSMART vector using T4 DNA ligase (New England Biolabs) according to the protocol of the PCR-SMART Cloning Kit (Lucigen, Middleton, WI, USA). The ligation mixture was transformed into DH5 $\alpha$ competent Escherichia coli cells (Invitrogen) by heat-shock transformation. Clones were screened by restriction enzyme digestion using EcoRV (a pSMART polycloning site flanking the inserted DNA sequence) and ScaI (which can be used to differentiate the Aallele (uncut) and the G-allele (cut) of SNP rs569356). Moreover, cloned OPRD1 promoter fragments were sequenced from both ends using two forward primers and two reverse primers. The forward primer SL1 (5'-CAGTCCAGTT ACGCTGGAGTC- $\left.3^{\prime}\right)$ and the reverse primer SR2 (5'-GGTCAGGTATGATTT AAATGGTCAGT- $3^{\prime}$ ) were included in the PCR-SMART Cloning Kit. An additional forward sequencing primer (Primer F2: 5'-TGGAATGGTTAG GTCTTGGA-3', homologous to OPRD1 upstream sequence from $-935 \mathrm{bp}$ to $-915 \mathrm{bp}$ ) and an additional reverse sequencing primer (Primer R2: 5'-GGAAG AGGATTCCGGATAAA- ${ }^{\prime}$, complementary to OPRD1 upstream sequence from -751 bp to $-731 \mathrm{bp}$ ) were synthesized. The location of the four primers and OPRD1 SNP rs569356 is depicted in Figure 1. Clones with the A-allele or the G-allele with otherwise identical sequences, and identical to the GenBank reference sequence (NT_004610.18) for OPRD1, were selected for construction of luciferase expression vectors.

The OPRD1 promoter fragment was excised from the pSMART clones using restriction enzyme EcoRV. Then it was phosphorylated and inserted into the SmaI cloning site in the promoterless and enhancerless pGL3-basic luciferase reporter vector (Promega, Madison, WI, USA) by blunt-end ligation. The OPRD1 promoter fragment was placed upstream of the firefly luciferase reporter gene. DNA was transformed into DH5 $\alpha$ competent E. coli cells (Invitrogen). The OPRD1 promoter sequence and cloning orientation in pGL3-basic vector were determined by restriction analysis and direct sequencing using primers F1, F2, R1 and R2 described above.

Transient transfection and dual-luciferase reporter assay HEK293 cells (purchased from the American Type Culture Collection or ATCC, Manassas, VA, USA) were grown in 48-well cell culture plates (Becton Dickinson, Franklin Lakes, NJ, USA; $3.5 \times 10^{5}$ cells per well). for $24 \mathrm{~h}$. They were then transiently transfected with a promoterless luciferase vector (pGL3basic) (Promega) or with a pGL3-basic vector with the OPRD1 promoter sequence harboring the rs569356 A-allele or G-allele (construct rs569356_Aallele or construct rs569356_G-allele) using linear polyethylenimine (molecular weight 25000) (Polysciences, Warrington, PA, USA) based on the method of Reed et al. ${ }^{21}$ An internal control vector pTK-RL, which contained the renilla luciferase gene downstream of a minimal HSV-TK promoter (Promega), was included in the transfection mix ( $375 \mathrm{ng}$ DNA in total, firefly construct/renilla construct $=10: 1$ ). Cells were harvested $48 \mathrm{~h}$ after transfection and luciferase assays were performed with the Dual Luciferase Assay Kit (Promega) according to the manufacturer's instructions. Cell lysate $(10 \mu \mathrm{l})$ were evaluated first for firefly luciferase activity and then for renilla luciferase activity in a white opaque 
96-well flat-bottom microplate (Becton Dickinson) using a DTX 880 Series Multimode Detector (Beckman Coulter, Fullerton, CA, USA). Firefly luciferase luminescence values were divided by renilla luciferase luminescence values from the same transfection to control for differences in transfection efficiency. For each transfection mix (pGL3+pTK-RL), the reporter assay was conducted four times in parallel.

\section{EMSA study}

EMSA studies were conducted to examine whether SNP rs569356 (A/G) influences the binding of human brain nuclear extract proteins (potentially containing OPRD1 transcription factors) to OPRD1 promoter sequence. Two double-stranded DNA oligonucleotides (21-bp) spanning OPRD1 promoter SNP rs569356 were synthesized. One contained rs569356 A-allele (5'-GTTTTA CACAATACTTTTTTG- $3^{\prime}$, the underlined is A-allele) and the other contained rs56935 G-allele (5'-GTTTTACACAGTACTTTTTTG- $3^{\prime}$, the underlined is G-allele). EMSA was performed on the basis of the instructions accompanying the DIG Gel Shift Kit, 2nd Generation (Roche, Mannheim, Germany). In brief, probes were first labeled with Digoxigenin-11-ddUTP at the $3^{\prime}$ end. A quantity of $2 \mu \mathrm{l}$ of labeled probes $\left(4 \mathrm{ng} \mu \mathrm{l}^{-1}\right)$ was then incubated with $6 \mu \mathrm{l}$ of human brain nuclear protein $\left(5 \mu \mathrm{gl}^{-1}\right)$ (Active Motif, Carlsbad, CA, USA). To prevent nonspecific binding of brain nuclear proteins to the probe, nonspecific competitors poly[d(I-C) $](1 \mu \mathrm{g})$ and poly-lysin $(0.1 \mu \mathrm{g})$ were added to the binding reaction ( $40 \mu \mathrm{l}$ of volume in total). To establish specificity, $4 \mu \mathrm{l}$ of unlabeled probes $\left(100 \mathrm{ng}^{-1} \mathrm{l}^{-1}\right)$ was added in the probe (labeled)-protein mixture as competitors. Following electrophoresis and gel blotting, the DIG-labeled probes were visualized by an enzyme immunoassay using an anti-Digoxigenin-alkaline phosphatase conjugate and the chemiluminescent substrate CSPD. An X-ray film was exposed to fluorescence for $15-25 \mathrm{~min}$ at room temperature and then developed. The image on the X-ray film was scanned at a resolution of $400 \mathrm{dpi}$. The density of the gel shift bands was quantified using software NIH ImageJ (the National Institutes of Health, Bethesda, MD, USA). ${ }^{22}$ The EMSA experiment was replicated once.

\section{Bioinformatics analysis}

We queried the OPRD1 promoter sequence harboring SNP rs569356 against the transcription factor database TRANSFAC, ${ }^{23}$ using the software MatInspector (Genomatix Software GmbH, München, Germany) ${ }^{24}$ to determine if the promoter SNP rs569356 is located in the binding site of potential transcription factors.

\section{Statistical analysis}

Mean luciferase activity corresponding to each construct was compared using the one-way analysis of variance in the SPSS 16.0 software package (SPSS, Chicago, IL, USA), and a one-step multiple comparison was performed using the Tukey Honestly Significant Difference post hoc test.

\section{RESULTS}

SNP rs569356 A-allele and G-allele differentially regulate OPRD1 promoter activity

Because the OPRD1 promoter region was subcloned into the pGL3 expression vector upstream to the firefly luciferase reporter gene using a blunt-end ligation, four possible expression vectors were obtained. As shown in Figure 2, two constructs (one with the A-allele and another with the G-allele) had the forward cloning orientation and another two constructs (one with the A-allele and another with the G-allele) had the reverse cloning orientation.

Before HEK293 cells were used for luciferase reporter gene assay, we verified that OPRD1 was expressed in these cells at a moderate level using the Affymetrix expression array study (Simen and Mane, 2009, unpublished observations). Thus, HEK293 cells express the necessary proteins to maintain $O P R D 1$ activity. For each of the five expression vectors (Constructs 1-4 and pGL3-basic, used as a negative control, see Figure 2), four independent reporter assays were conducted in replication. The raw data are presented in Supplementary material Table S1. To adjust for variation in transfection efficiency and cell density, the firefly fluorescence reading was normalized by the renilla fluorescence reading (as a ratio of firefly to renilla) (Figure 3). A one-way analysis of variance revealed a main effect of constructs (pGL3-basic vector vs A-allele_forward vector vs G-allele_forward vector: $\left.\mathrm{F}_{(2,11)}=179.37, P<0.001\right)$. Both the A-allele_forward and the G-allele_forward constructs (with OPRD1 promoter sequence in the correct orientation) showed considerably higher luciferase activity
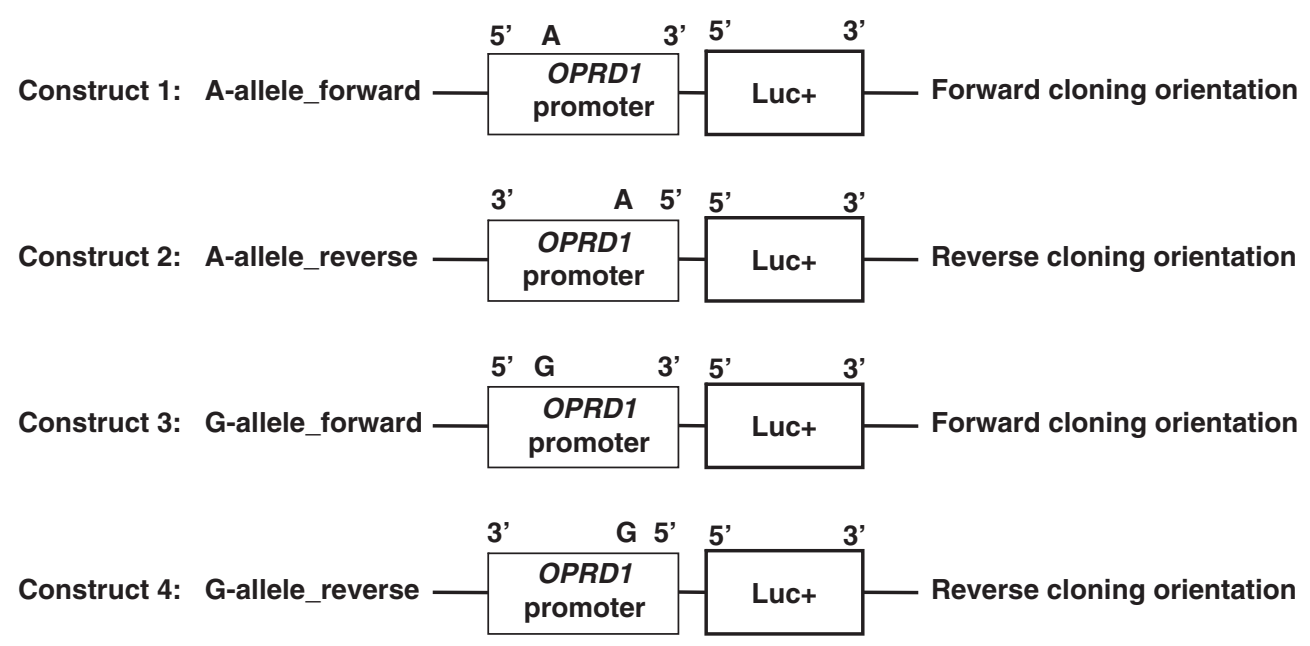

pGL3-basic vector

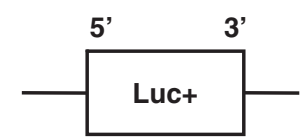

Figure 2 Construction of four pGL3 expression vectors. OPRD1 promoter fragment containing SNP rs569356 A-allele or G-allele was excised from a pSMART plasmid vector and subcloned into a pGL3 expression vector by blunt-end ligation. OPRD1 promoter sequence was placed upstream to the firefly luciferase reporter gene (luc+). 


\section{$P<0.001$}

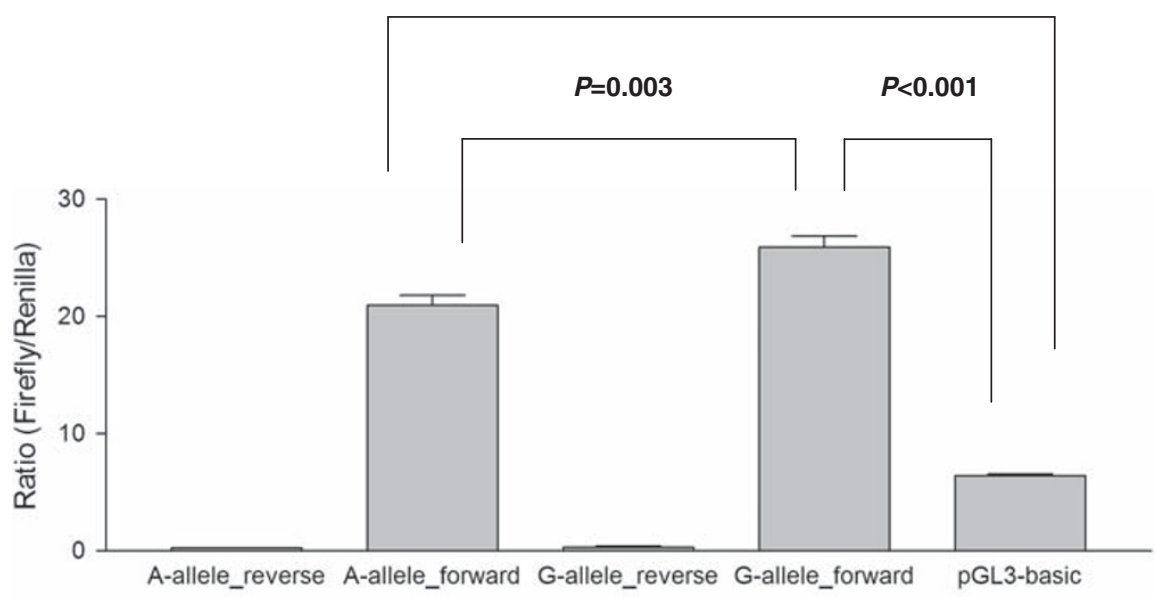

Five Expression Vectors

Figure 3 Luciferase reporter gene assay of the effect of SNP rs569356 (A/G) on OPRD1 promoter activity. Four constructed vectors (A-allele_reverse, Aallele_forward, G-allele_reverse and G-allele_forward) and the pGL3-basic vector were co-transfected respectively with pTK-RL (internal control vector harboring renilla luciferase gene) into HEK293 cells. Cell lysate was used to measure sequentially the activity of firefly luciferase and renilla luciferase. Firefly luminescence was normalized by renilla luminescence. Four independent replications of the experiment were performed. The difference in firefly luciferase activity between different vectors was analyzed by one-way analysis of variance and the Tukey Honestly Significant Difference test. $Y$ axis: ratios of firefly fluorescence to renilla fluoresence; $X$ axis: five expression vectors.

(A-allele_forward vector vs pGL3-basic vector: $P<0.001$; G-allele forward vector vs pGL3-basic vector: $P<0.001)$ compared with the promoterless pGL3-basic vector (by the Tukey Honestly Significant Difference test). These results support the conclusion that the 2250-bp region upstream of OPRD1 that we cloned possesses strong promoter activity. When the OPRD1 promoter sequence was placed in the opposite orientation, firefly luciferase activity was suppressed (Figure 3). In the forward orientation, the G-allele construct was associated with significantly higher luciferase activity than the A-allele construct $(P=0.003)$ (by the Tukey Honestly Significant Difference test), suggesting that the A to G polymorphism at position $-1968 \mathrm{bp}$ of the OPRD1 regulatory region increases the activity of the OPRD1 promoter.

\section{Binding of brain nuclear proteins to OPRD1 promoter sequence harboring rs569356}

Because the G-allele construct was associated with significantly higher luciferase activity, we hypothesized that the G-allele might bind to certain activating transcription factors more effectively than the Aallele. Double-stranded DNA probes encoding the OPRD1 rs569356 A-allele or G-allele were labeled with digoxigenin and mixed with a human brain nuclear extract in binding buffer. As shown in Figure 4a, human brain nuclear proteins retarded the migration of probes for both the G-allele (lane 5) and the A-allele (lane 8). The interaction could be inhibited by competition with unlabeled probe for the Gallele (lane 6) or the A-allele (lane 9). Moreover, both the G-allele probe and the A-allele probe showed two gel shift bands of similar size. As the two gel shift bands for the G-allele probe or the A-allele probe were very close, their density was measured together. As shown in Figure $4 \mathrm{~b}$, the density of the gel shift band for the G-allele probe is 1.61 times higher than that of the A-allele probe. In addition, a probe for Oct $2 \mathrm{a}$ and purified Oct $2 \mathrm{a}$ protein (Figure $4 \mathrm{a}$, lanes 1-3) were included as a positive control to test the quality of the reagents. The EMSA replication results are shown in Supplementary material Figure S1 and
Table S2. The mean ratio ( \pm s.d.) of the G-allele gel shift band density to the A-allele gel shift band density was $1.64( \pm 0.04)$.

\section{Bioinformatics analysis results}

The A-allele of SNP 569356 was in the binding site of two transcription factors (Fork head homologous $\mathrm{X}$ binds DNA with a dual sequence specificity (FHXA and FHXB): ttttacACAAtactttt; SWI/ SNF related, matrix associated, actin-dependent regulator of chromatin, subfamily a, member 3 (Smarca3): caatACTTttt)). The G-allele of SNP rs569356 was in the binding site of three transcription factors (NK3 homeobox 1 (NKX3-1): tttacacaGTACttttttg; zinc-finger proliferation 1 (Zipro 1): cAGTAct; Smarca3: cagtACTTttt) (The underlining shows the allele (A or $\mathrm{G}$ ) and capital letters represent the core binding site).

\section{DISCUSSION}

We used both the luciferase reporter gene assay and EMSA to examine the function of OPRD1 promoter SNP rs569356. Our findings indicate that SNP rs569356 (A/G) affects the activity of the OPRD1 promoter. Luciferase reporter assays demonstrated that the 2250-bp fragment upstream of OPRD1 possesses strong promoter activity. OPRD1 promoter region with either rs569356 A-allele or G-allele can drive reporter gene expression at a substantial level (Figure 3). Moreover, significantly higher luciferase activity was found for the rs569356 Gallele in comparison with the A-allele. This finding suggests that SNP rs569356 is functional and the substitution of a $G$ for an A can enhance OPRD1 promoter activity, possibly by enhancing transcription factor binding.

Although we are unable to determine whether OPRD1 promoter activity observed in HEK293 cells (derived from human embryonic kidney cells) reflects promoter activity in neural cells, microarray results demonstrated that OPRD1 is expressed in HEK293 cells at a moderate level. Thus, transcription factors required for OPRD1 transcription are expressed in HEK293 cells and the observed 


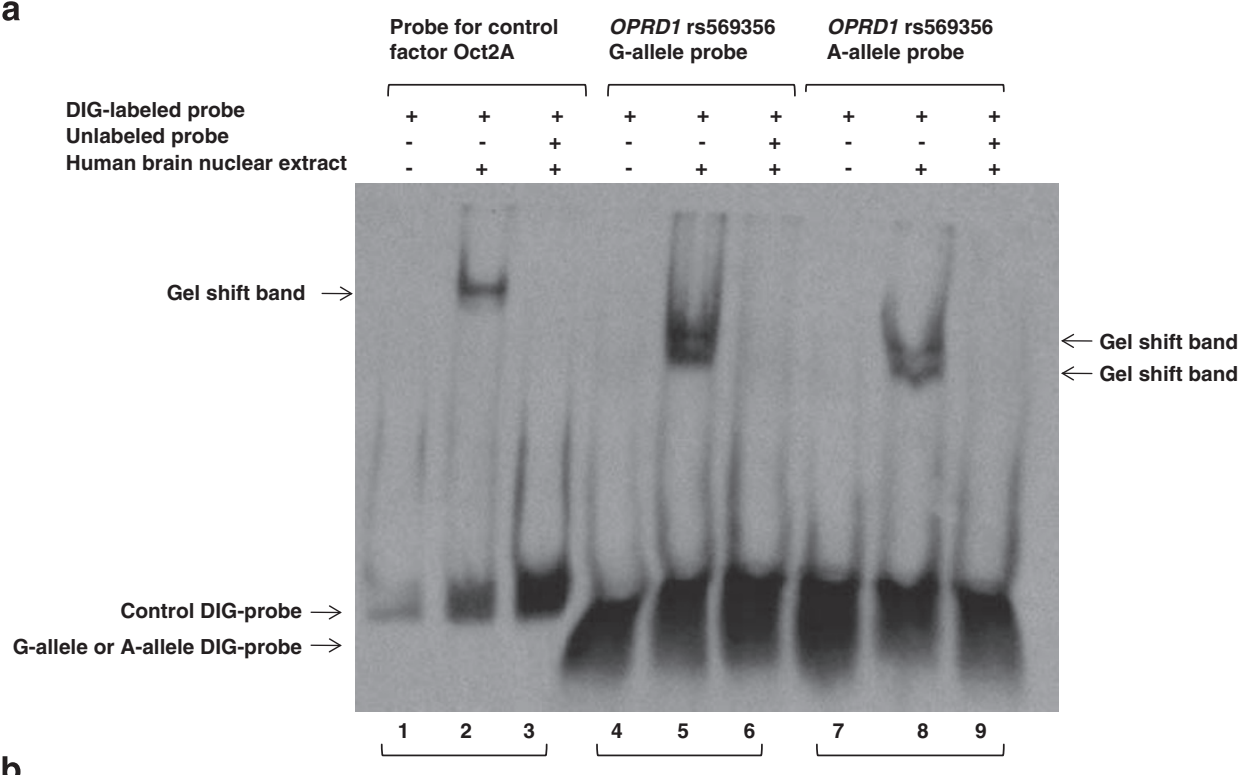

b
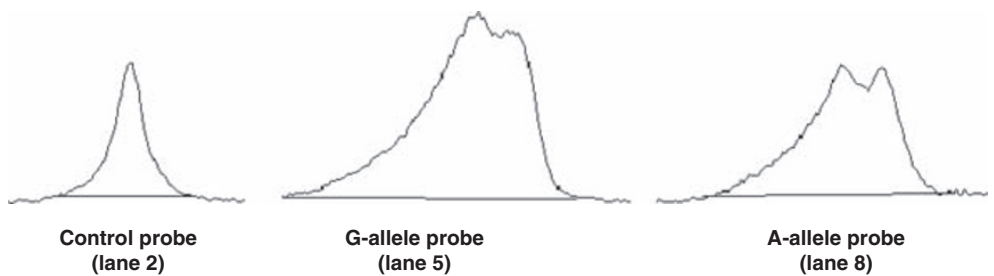

Figure 4 Electrophoretic mobility shift assay (EMSA). (a) The binding of human brain nuclear proteins (potentially containing transcription factors) with OPRD1 promoter sequence (harboring either the rs569356 A-allele or G-allele) was determined by the EMSA. Lanes 1-3: control probe and control factor Oct2A included in the DIG Gel Shift Kit (Roche). Lanes 4-6: OPRD1 rs569356 G-allele probe. Lanes 7-9: OPRD1 rs569356 A-allele probe. Lanes 1, 4 and 7: in the absence of brain nuclear extract, DIG-labeled probes showed no retardation; lanes 2, 5 and 8: in the presence of brain nuclear extract, DIGlabeled probes showed retardation, and gel shift bands due to the slow migration of protein-probe complex were observed; lanes 3, 6 and 9: in the presence of brain nuclear extract and unlabeled probes (as competitors), DIG-labeled probes showed no retardation. (b) The intensity of gel shift bands derived from the control probe (lane 2), the G-allele probe (lane 5) and the A-allele probe (lane 8) was scanned at $400 \mathrm{dpi}$ and quantified by software ImageJ.22

effects of rs569356 likely reflect OPRD1 promoter activity in neuronal cells. In this study, we only tested OPRD1 promoter activity under basal unstimulated conditions. It is possible that additional effects of rs569356 are evident when promoter activity is stimulated; for example, under conditions of elevated protein kinase A activity.

The results from our EMSA assays, which were conducted with human brain nuclear proteins, paralleled those observed from our promoter assays. EMSA studies revealed that human brain nuclear proteins interact with probes containing OPRD1 promoter sequence with either the A-allele or the G-allele of SNP rs569356. Both the A-allele and the G-allele probes showed two gel shift bands, but the binding of the G-allele probe to nuclear proteins was $1.64 \pm 0.04$ (mean \pm s.d.) times that of the A-allele probe (Figure 4, Supplementary Figure S1 and Table S2). The enhanced DNA-protein binding may be responsible for the increased OPRD1 promoter activity that we observed in our promoter assays. Consistent with the observation that SNP rs569356 only slightly modifies the binding of transcription factors to OPRD1 promoter sequence, both the Aallele construct and the G-allele construct showed high expression levels of the reporter gene. However, differential expression levels associated with these two alleles were observed as well (Figure 3).

Bioinformatics analysis revealed that SNP rs569356 may affect the binding of a number of transcription factors including FHXA and
FHXB, Smarca3, NKX3-1 and Zipro 1. In this study, we did not use super-gel shift assays to verify these interactions because antibodies specific for them are unavailable. Future studies could use immunoprecipitation plus massively parallel DNA sequencing (ChIP-Seq) to test whether these transcription factors are involved in the formation of transcriptional complexes at the rs569356 site.

Functional characterization of gene variants is important for interpreting the genetic mechanism of diseases. SNPs have been widely used in candidate gene and genome-wide association studies to understand the genetic basis of complex disorders. However, the biological relevance from SNP analyses is not easily interpretable. Thus, it is critical to study the function of disease-associated genetic variants. Previous studies have demonstrated that opioid receptor availability is positively correlated with levels of craving for abused substances. ${ }^{25-27}$ These studies have also shown significant regional increases in opioid receptor availability in early abstinence from dependence on cocaine, alcohol or opioids when compared with controls. Thus, elevated OPRD1 expression levels may increase the rewarding effect of drugs of abuse, leading to increased vulnerability to substance dependence (including opioid dependence). The finding from this study that the minor G-allele of SNP rs569356 increases OPRD1 promoter activity supports our previous genetic association results that the minor G-allele is a genetic risk factor for opioid dependence. 
Our findings may have important implications for the pharmacotherapy of substance dependence. Lowering the expression, activity or occupancy of the $\delta$-opioid receptor in patients with substance dependence may facilitate recovery from dependence. In fact, nonselective opioid receptor antagonists such as naltrexone (with a high affinity for the $\mu$ - and $\kappa$-opioid receptors but a low affinity for the $\delta$-opioid receptor) and naloxone (with an extremely high affinity for the $\mu$-opioid receptors but a low affinity for the $\delta$ - and $\kappa$-opioid receptors) have been used in the management of alcohol and/or opioid dependence. ${ }^{28}$ It is possible that $\delta$-opioid receptor antagonists or drugs that downregulate $\delta$-opioid receptor expression will show efficacy in the treatment of substance dependence. Animal studies have provided some support for this hypothesis. Nielsen et al. ${ }^{29}$ found that a novel $\delta$-opioid receptor antagonist SoRI-9409, a derivative of naltrexone and with a high affinity for the $\delta$-opioid receptor, produced a selective and long-lasting decrease in ethanol consumption in heavy drinking rats. A recent study also demonstrated that the $\delta$-opioid receptor antagonists naltrindole and naltriben prevented the enhanced response to the conditioned rewarding effects of morphine in rats. ${ }^{30}$ Given that SNP rs569356 influences OPRD1 promoter activity and possibly OPRD1 expression levels, future pharmacogenetic studies need to take into consideration its genetic effect on the response to treatment of substance dependence with $\delta$-opioid receptor antagonists.

Promoter activity may be regulated by several independent or correlated variants. For example, Zhao et al. ${ }^{31}$ studied the association of variants in the promoter region of the Chitinase 3-like 1gene (CHI3L1) and schizophrenia. They found that an at-risk haplotype showed low promoter activity but a protective haplotype showed high promoter activity, and these haplotypes consisted of alleles of three correlated loci in the promoter region. Although SNP rs569356 is the only common variant identified so far, we cannot exclude the possibility that rare variants in the OPRD1 promoter region may exert an even larger effect on OPRD1 transcription than SNP rs569356. Therefore, deep sequencing of OPRD1 promoter for rare variants is necessary.

Taken together, this study demonstrated that SNP rs569356 is a functional variant that alters OPRD1 promoter activity. Although OPRD1 promoter SNP rs569356 and OPRD1 non-synonymous SNP C80T (in exon 1) are highly linked, they are likely to be two independent genetic variants, which influence the vulnerability to opioid dependence through two different mechanisms. The former one may affect promoter activity and thus compromise OPRD1 transcription (as shown in this study). The latter one may directly change $\delta$-opioid receptor activity by changing the amino-acid sequence of the receptor protein. We are now in the process of investigating the function of the non-synonymous variant C80T by receptor binding assays. In addition, future pharmacotherapy of opioid dependence must consider the effect of these two OPRD1 variants on the outcome of treatment.

\section{CONFLICT OF INTEREST}

Dr Kranzler has received consulting fees from Ortho-McNeil Pharmaceuticals (Raritan, NJ, USA), H. Lundbeck A/S (Copenhagen, Denmark), Forest Pharmaceuticals (St Louis, MO, USA), elbion NV (Leuven, Belgium), Sanofi-Aventis (Bridgewater, NJ, USA), Solvay Pharmaceuticals (Brussels, Belgium) and Alkermes (Cambridge, MA, USA). He has received research support from Ortho-McNeil Pharmaceuticals, Bristol-Myers Squibb Company (New York, NY, USA) and Merck (Whitehouse Station, NJ, USA), and honoraria from Forest
Pharmaceuticals and Alkermes. Other authors have no conflict of interest to report.

\section{ACKNOWLEDGEMENTS}

Kiran Patel provided excellent technical assistance with the luciferase reporter gene assays. HZ was supported by the National Institute of Drug Abuse (NIDA) Grant K99/R00DA022891 and a research award from the Alcoholic Beverage Medical Research Foundation (ABMRF). JRG was supported by the National Institute of Neurological Disorders and Stroke (NINDS) Grant R01NS043530. AAS was supported by the National Institute of Aging (NIA) Grant AG030970 and by the Claude D Pepper Older Americans Independence Center at Yale University School of Medicine. This study was also supported by NIDA Grants R01 DA12690 and R01 DA12849, K12 DA000167 and the VA CT MIRECC Center to JG

1 Stengaard-Pedersen, K. Comparative mapping of opioid receptors and enkephalin immunoreactive nerve terminals in the rat hippocampus. A radiohistochemical and immunocytochemical study. Histochemistry 79, 311-333 (1983).

2 Bodnar, R. J. Endogenous opiates and behavior: 2006. Peptides 28, 2435-2513 (2007).

3 Corbett, A. D., Henderson, G., McKnight, A. T. \& Paterson, S. J. 75 years of opioid research: the exciting but vain quest for the Holy Grail. Br. J. Pharmacol. 147, S153-S162 (2006).

4 Spanagel, R., Herz, A. \& Shippenberg, T. S. Opposing tonically active endogenous opioid systems modulate the mesolimbic dopaminergic pathway. Proc. Natl Acad. Sci. USA 89, 2046-2050 (1992).

5 Shippenberg, T. S., Lefevour, A. \& Heidbreder, C. kappa-Opioid receptor agonists prevent sensitization to the conditioned rewarding effects of cocaine. J. Pharmacol. Exp. Ther. 276, 545-554 (1996).

6 Herz, A. Endogenous opioid systems and alcohol addiction. Psychopharmacology (Berl) 129, 99-111 (1997).

7 Zhu, Y., King, M. A., Schuller, A. G., Nitsche, J. F., Reidl, M., Elde, R. P., et al. Retention of supraspinal delta-like analgesia and loss of morphine tolerance in delta opioid receptor knockout mice. Neuron 24, 243-252 (1999).

8 Uhl, G. R., Childers, S. \& Pasternak, G. An opiate-receptor gene family reunion. Trends. Neurosci. 17, 89-93 (1994).

9 Mayer, P., Rochlitz, H., Rauch, E., Rommelspacher, H., Hasse, H. E., Schmidt, S., et al. Association between a delta opioid receptor gene polymorphism and heroin dependence in man. Neuroreport 8, 2547-2550 (1997)

10 Gelernter, J. \& Kranzler, H. R. Variant detection at the delta opioid receptor (OPRD1) locus and population genetics of a novel variant affecting protein sequence. Hum. Genet. 107, 86-88 (2000).

11 Franke, P., Nothen, M. M., Wang, T., Neidt, H., Knapp, M., Lichtermann, D., et al. Human delta-opioid receptor gene and susceptibility to heroin and alcohol dependence. Am. J. Med. Genet. 88, 462-464 (1999).

12 Xu, K., Liu, X. H., Nagarajan, S., Gu, X. Y. \& Goldman, D. Relationship of the deltaopioid receptor gene to heroin abuse in a large Chinese case/control sample. Am. J. Med. Genet. 110, 45-50 (2002).

13 Loh, eW., Fann, C. S., Chang, Y. T., Chang, C. J. \& Cheng, A. T. Endogenous opioid receptor genes and alcohol dependence among Taiwanese Han. Alcohol. Clin. Exp. Res. 28, 15-19 (2004).

14 Zhang, H., Kranzler, H. R., Yang, B. Z., Luo, X. \& Gelernter, J. The OPRD1 and OPRK1 loci in alcohol or drug dependence: OPRD1 variation modulates substance dependence risk. Mol. Psychiatry 13, 531-543 (2008).

15 De La Vega, F. M., Isaac, H. I. \& Scafe, C. R. A tool for selecting SNPs for association studies based on observed linkage disequilibrium patterns. Pac. Symp. Biocomput 11, 487-498 (2006).

16 Orphanides, G. \& Reinberg, D. A unified theory of gene expression. Cell 108, 439-451 (2002).

17 Tournamille, C., Colin, Y., Cartron, J. P. \& Le Van, K. C. Disruption of a GATA motif in the Duffy gene promoter abolishes erythroid gene expression in Duffy-negative individuals. Nat. Genet. 10, 224-228 (1995).

18 Knight, J. C., Udalova, I., Hill, A. V., Greenwood, B. M., Peshu, N., Marsh, K., et al. A polymorphism that affects OCT-1 binding to the TNF promoter region is associated with severe malaria. Nat. Genet. 22, 145-150 (1999).

19 Udalova, I. A., Richardson, A., Denys, A., Smith, C., Ackerman, H., Foxwell, B., et al. Functional consequences of a polymorphism affecting NF-kappaB p50-p50 binding to the TNF promoter region. Mol. Cell Biol. 20, 9113-9119 (2000).

20 Farzaneh-Far, A., Davies, J. D., Braam, L. A., Spronk, H. M., Proudfoot, D., Chan, S. W., et al. A polymorphism of the human matrix gamma-carboxyglutamic acid protein promoter alters binding of an activating protein-1 complex and is associated with altered transcription and serum levels. J. Biol. Chem. 276, 32466-32473 (2001)

21 Reed, S. E., Staley, E. M., Mayginnes, J. P., Pintel, D. J. \& Tullis, G. E. Transfection of mammalian cells using linear polyethylenimine is a simple and effective means of 
producing recombinant adeno-associated virus vectors. J. Virol. Methods 138, 85-98 (2006).

22 Collins, T. J. Image J for microscopy. Biotechniques 43, 25-30 (2007).

23 Quandt, K., Frech, K., Karas, H., Wingender, E. \& Werner, T. MatInd and MatInspector: new fast and versatile tools for detection of consensus matches in nucleotide sequence data. Nucleic Acids Res. 23, 4878-4884 (1995).

24 Cartharius, K., Frech, K., Grote, K., Klocke, B., Haltmeier, M., Klingenhoff, A., et al. MatInspector and beyond: promoter analysis based on transcription factor binding sites. Bioinformatics 21, 2933-2942 (2005)

25 Heinz, A., Reimold, M., Wrase, J., Hermann, D., Croissant, B., Mundle, G., et al. Correlation of stable elevations in striatal mu-opioid receptor availability in detoxified alcoholic patients with alcohol craving: a positron emission tomography study using carbon 11-labeled carfentanil. Arch. Gen. Psychiatry 62, 57-64 (2005).

26 Gorelick, D. A., Kim, Y. K., Bencherif, B., Boyd, S. J., Nelson, R., Copersino, M., et al. Imaging brain mu-opioid receptors in abstinent cocaine users: time course and relation to cocaine craving. Biol. Psychiatry 57, 1573-1582 (2005).
27 Williams, T. M., Davies, S. J., Taylor, L. G., Daglish, M. R., Hammers, A., Brooks, D. J., et al. Brain opioid receptor binding in early abstinence from alcohol dependence and relationship to craving: an [11C]diprenorphine PET study. Eur. Neuropsychopharmacol. 19, 740-748 (2009).

28 Sinclair, J. D. Evidence about the use of naltrexone and for different ways of using it in the treatment of alcoholism. Alcohol Alcohol. 36, 2-10 (2001).

29 Nielsen, C. K., Simms, J. A., Pierson, H. B., Li, R., Saini, S. K., Ananthan, S., et al. A novel delta opioid receptor antagonist, SoRI-9409, produces a selective and long-lasting decrease in ethanol consumption in heavy-drinking rats. Biol. Psychiatry 64, 974-981 (2008).

30 Shippenberg, T. S., Chefer, V. I. \& Thompson, A. C. Delta-opioid receptor antagonists prevent sensitization to the conditioned rewarding effects of morphine. Biol. Psychiatry 65, 169-174 (2009).

31 Zhao, X., Tang, R., Gao, B., Shi, Y., Zhou, J., Guo, S., et al. Functional variants in the promoter region of Chitinase 3 -like $1(\mathrm{CHI} 3 \mathrm{~L} 1)$ and susceptibility to schizophrenia. Am. J. Hum. Genet. 80, 12-18 (2007).

Supplementary Information accompanies the paper on Journal of Human Genetics website (http://www.nature.com/jhg) 